\title{
SMARCC2 combined with c-Myc inhibits the migration and invasion of glioma cells via modulation of the Wnt/B-catenin signaling pathway
}

\author{
CHIYANG LI ${ }^{1}$, CHENGSHUO FEI $^{1}$, JUNJIE LI ${ }^{1}$, HANG WU $^{2}$, LEI CHEN $^{1}$, RAMZI ROSHANI $^{1}$, HONG LI $^{1}$, \\ LINYONG SHI ${ }^{1}$, CHONG SONG ${ }^{1}$, JUNWEI GU ${ }^{1}$, YUNTAO LU ${ }^{1,3,4}$ and QIANG ZHOU ${ }^{1,3,4}$ \\ Departments of ${ }^{1}$ Neurosurgery and ${ }^{2}$ Hematology; ${ }^{3}$ Nanfang Neurology Research Institution, Nanfang Hospital, \\ Southern Medical University; ${ }^{4}$ Nanfang Glioma Centre, Guangzhou, Guangdong 510515, P.R. China
}

Received July 29, 2020; Accepted March 19, 2021

DOI: $10.3892 / \mathrm{mmr} .2021 .12190$

\begin{abstract}
Glioma is the most common type of central nervous system tumor. SWItch/sucrose non-fermentable (SWI/SNF) is a tumor suppressor that serves an important role in epithelial-mesenchymal transition (EMT). The present study aimed to identify key molecules involved in the EMT process. SWI/SNF related, matrix associated, actin dependent regulator of chromatin subfamily c member 2 (SMARCC2) is mutated in and its expression is low in multiple types of cancer. SMARCC2 is the core subunit of the chromatin-remodeling complex, SWI/SNF. Relative mRNA SMARCC2 expression levels in human glioma tissue were analyzed via reverse transcription-quantitative PCR, whereas the protein expression levels were determined via immunohistochemistry staining. SMARCC2 expression was knocked down in glioma cells using small interfering RNA (si) and overexpressed by infection with adenovirus vectors carrying SMARCC 2 cDNA. Wound healing and Transwell assays were performed to assess cell migration and invasion, respectively. Subsequently, immunofluorescence and western blotting were performed to analyze the expression levels of the oncogene c-Myc, which is associated with SMARCC2. SMARCC2 combines with C-MYC to downregulate its expression. Consistent with the results of the bioinformatics analysis, which revealed that the upregulated expression levels of SMARCC2 were associated with a more favorable prognosis in patients with glioma, the mRNA and protein expression levels of SMARCC2 were significantly
\end{abstract}

Correspondence to: Dr Qiang Zhou or Professor Yuntao Lu, Department of Neurosurgery, Nanfang Hospital, Southern Medical University, 1838 Guangzhou Avenue North, Guangzhou, Guangdong 510515, P.R. China

E-mail: ellsonchou@163.com

E-mail: 947804460@qq.com

Key words: SWItch/sucrose non-fermentable, SWI/SNF related, matrix associated, actin dependent regulator of chromatin subfamily c member 2, c-Myc, epithelial-mesenchymal transition, glioma upregulated in low-grade glioma tissues compared with high-grade glioma tissues. The results of the wound healing assay demonstrated that cell migration was significantly increased in the siSMARCC2-1/3 groups compared with the negative control (NC) group. By contrast, the migratory ability of cells was significantly reduced following transduction with adenovirus overexpressing SMARCC2, which upregulated the expression of SMARCC2, compared with the lentiviral vector-non-specific control (LVS-NC) group. The Transwell assay results further showed that SMARCC2 overexpression significantly inhibited the migratory and invasive abilities of U87MG and LN229 cells compared with the LVS-NC group. Co-immunoprecipitation assays were subsequently conducted to validate the binding of SMARCC2 and c-Myc; the results demonstrated that the expression of c-Myc was downregulated in adenovirus-transfected cells compared with LVS-NC-transfected cells. The results of the western blotting experiments demonstrated that the expression levels of $\mathrm{N}$-cadherin, vimentin, snail family transcriptional repressor 1 and $\beta$-catenin were notably downregulated, whereas the expression levels of T-cadherin were markedly upregulated in cell lines stably overexpressing SMARCC2 compared with the LVS-NC group. In conclusion, the results of the present study suggested that SMARCC2 may inhibit Wnt/ $\beta$-catenin signaling by regulating $\mathrm{c}-\mathrm{Myc}$ expression in glioma. SMARCC2 regulates the EMT status of the glioblastoma cell line by mediating the expression of the oncogene C-MYC to inhibit its migration and invasion ability. Thus, SMARCC2 may function as a tumor suppressor or oncogene by regulating associated oncogenes or tumor suppressor genes.

\section{Introduction}

ATP-dependent chromatin-remodelling complexes (remodelers), SWItch/sucrose non-fermentable (SWI/SNF), were reported to serve a crucial role in gene regulation, where they affected numerous biological processes (1). However, the molecular architecture of SWI/SNF complexes was not studied in detail until the previous 5 years (2-4). Nonetheless, to the best of our knowledge, the role of their core subunit, SWI/SNF related, matrix associated, actin dependent regulator 
of chromatin subfamily c member 2 (SMARCC2), in the development and progression of glioma remains poorly understood. Over the past two decades, there have been rapid developments in research regarding chromatin structures that govern crucial cellular processes, including DNA replication, transcription and post-transcriptional gene regulation (5). The mammalian SWI/SNF family of chromatin remodelers, BAF nuclear assembly factor 1 (BAF) and polybromo-associated BAF, regulate chromatin structure and transcription via sliding, ejecting and reconstituting the nucleosomes, and mutations in each have previously been associated with numerous cancer types, such as breast, colon, liver and stomach cancer (6-10). Among the four core subunits of the SWI/SNF complex [SMARC subfamily a member 4 (SMARCA4), BAF155, SMARCC2 and SMARC subfamily b member 1], SMARCC2 forms a part of the SWI/SNF base module that displays a compact fold and can be divided into five closely associated submodules: The head, thumb, palm, bridge and fingertips. The thumb is formed by the SANT domain of SMARCC2, the pre-HSA of SMARCA4 and the C-terminal helices of SMARC subfamily d member 1 (2).

Glioma is the most common type of primary malignant tumor of the central nervous system and is strongly resistant to postoperative radiotherapy and chemotherapy (11-13). Glioblastoma (GBM) is known to be the most severe type (level 4) of glioma owing to its malignant nature. As the standard Stupp therapy for glioblastoma, the most common type of intracranial aggressive tumor, ionizing radiation plus concomitant and adjuvant temozolomide is widely used following surgical resection. Temozolomide triggers autophagyassociated cell death to inhibit tumor growth in GBM (3). However, the prognosis of patients with GBM remains poor, with a median survival of 14.6 months. Numerous previous studies focused on identifying potential molecular targeted therapies and associated molecular pathways involved in GBM carcinogenesis have shed light on its pathogenesis, which has improved patient prognosis (14-18).

The present study aimed to investigate the antitumor effects of SMARCC2 on glioma cells. Furthermore, the possible underlying mechanisms associated with the effects of SMARCC2 were also investigated.

\section{Materials and methods}

Patient studies. In total, 62 patients (age, 21-67 years; 42 males and 20 females) with newly diagnosed GBM who had undergone surgery plus standard chemoradiotherapy (Stupp regimen) were recruited from October 2012 to March 2019. Patients had no chronic disease, such as cardiovascular disease, hypertension and hyperglycemia. All glioma tissue specimens were collected from Nanfang Hospital of Southern Medical University (Guangzhou, China). Fresh samples were stored in $-196^{\circ} \mathrm{C}$ liquid nitrogen following surgical removal. All patients provided written informed prior to participation in the study, and all study results were stored and analyzed anonymously. The present study protocol was approved by the Institutional Review Board at Nanfang Hospital of Southern Medical University (approval no. 81772656). All research was performed in accordance with the principles of the Declaration of Helsinki of 1975. Tumor tissue obtained from the patients was graded according to the World Health Organization (19) criteria.

Cell lines and culture. U87MG (cat. no. CC-Y1528), which are most probably a glioblastoma cell line of unknown origin, T98G and LN229 cell lines were purchased from American Type Culture Collection. Cells were cultured in DMEM (containing $4.5 \mathrm{~g} / 1$ glucose; cat. no. 11995065) supplemented with 10\% FBS (cat. no. 16140071) and puromycin (all Gibco; Thermo Fisher Scientific, Inc.), and maintained in a humidified incubator at $37^{\circ} \mathrm{C}$ in a $5 \% \mathrm{CO}_{2}$ atmosphere.

Cell transfection and transduction. The nucleotide sequences of small interfering RNAs (siRNAs/sis) targeting SMARCC2 were synthesized by Shanghai GenePharma Co., Ltd. and were as follows: siSMARCC2-1 forward, 5'-CUCGGCAAGAACUAC AAGATT-3' and reverse, 5'-UCUUGUAGUUCUUGCCGA GTT-3'; and siSMARCC2-3 forward, 5'-GGCGUUACGAUU UCCAGAATT-3' and reverse, 5'-UUCUGGAAAUCGUAA CGCCTT-3'. An NC siRNA was also used. The siRNA negative control sequence was as follows: 5'-TTUUGAACCAAGAAG CCUCCC-3' (Shanghai GenePharma Co., Ltd.). To overexpress SMARCC2, SMARCC2 cDNA was subcloned into an adenovirus expression vector, which also encoded GFP (Shanghai GenePharma Co., Ltd.), namely LVS-SMARCC2 (overexpression; oe). Lentiviral vector non-specific control (LVS-NC) was used as the control. The U87MG and LN229 glioma cells were seeded $24 \mathrm{~h}$ prior to transfection at room temperature, at 50-60\% confluence, then transfected using Lipofectamine ${ }^{\circledR}$ 2000 (Invitrogen; Thermo Fisher Scientific, Inc.) according to the manufacturer's protocol. The cells were transfected with $50 \mathrm{nM}$ siRNA and $2.5 \mu \mathrm{g}$ plasmid at room temperature, according to the manufacturer's protocol. The transduction efficiency was analyzed by the intensity of GFP fluorescence at $48 \mathrm{~h}$ post-transduction. A confocal microscope (Olympus CX23; Olympus Corporation) under x 200 magnification was used to observe the fluorescence intensity of the cells. Subsequent experiments were performed $48 \mathrm{~h}$ post-transfection.

Reverse transcription-quantitative PCR (RT-qPCR). Total RNA was extracted from transfected cells using TRIzol ${ }^{\circledR}$ (Invitrogen; Thermo Fisher Scientific, Inc.) according to the manufacturer's protocol. Total RNA $(1 \mu \mathrm{g})$ was reverse transcribed into cDNA according to the reverse transcription kit (RevertAid First Strand cDNA synthesis kit) (Takara Bio, Inc.) protocol and genomic DNA was also removed using the gDNA Eraser from the kit. qPCR was subsequently performed using SYBR Premix Ex Taq II (Takara Bio, Inc.) on a StepOne ${ }^{\mathrm{TM}}$ Real-Time PCR system (Applied Biosystems; Thermo Fisher Scientific, Inc.). The following primer sequences were used for qPCR: SMARCC2 forward, 5'-ACTGCCGATCAAATG TTTCCT-3' and reverse, 5'-ACAGGCAATTATTCTGCA CCAAG-3'; and GAPDH forward, 5'-AGAAGGCTGGGG CTCATTTG-3' and reverse, 5'-AGGGGCCATCCACAGTCT TC-3'. The following thermocycling conditions were used for qPCR: Initial denaturation at $95^{\circ} \mathrm{C}$ for $5 \mathrm{~min}$; followed by 40 cycles at $95^{\circ} \mathrm{C}$ for $15 \mathrm{sec}$ and $60^{\circ} \mathrm{C}$ for $30 \mathrm{sec}$. The relative expression levels of SMARCC2 were quantified using the $2^{-\triangle \Delta C q}$ method and normalized to GAPDH, which served as the endogenous control (20). 
Western blotting. The cells were harvested and the total protein was extracted using RIPA lysis buffer with protease-phosphatase inhibitor (EMD Millipore). Pierce BCA Protein assay kit (Thermo Fisher Scientific, Inc.) was used to quantify protein expression. The proteins $(30 \mu \mathrm{g} /$ lane $)$ were separated via $8-10 \%$ SDS-PAGE, transferred onto PVDF membranes and blocked with $0.02 \mathrm{M}$ TBS containing 5\% BSA (Abcam) and $0.1 \%$ Tween-20 for $2 \mathrm{~h}$ at room temperature. The membranes were subsequently incubated overnight at $4^{\circ} \mathrm{C}$ with the following primary antibodies (all from Abcam): Rat anti-SMARCC2 (1:1,000; cat. no. ab243634), rat anti-c-Myc (1:1,000; cat. no. ab32072), anti-tumor protein D52 (TPD52) like 2 (TPD52L2; 1:1,000; cat, no. ab234819), anti-snail family transcriptional repressor 1 (Snail; 1:1,000; cat. no. no. ab216347), rat anti-N-cadherin, rat anti-T-cadherin $(1: 1,000$; cat. no. ab167407), rabbit anti- $\beta$-catenin (1:1,000; cat. no. ab32572), anti-vimentin $(1: 1,000$; cat. no. ab92547), anti-GAPDH $(1: 3,000$; cat. no. ab8245) and mouse anti- $\beta$-actin $(1: 3,000$; cat. no. ab8226). Subsequently, horseradish peroxidase-conjugated secondary goat anti-rabbit IgG H\&L (1:5,000; cat. no. b6721; Abcam), was incubated with the membrane at room temperature for $1 \mathrm{~h}$. The membranes were visualized using an ECL kit (Bio-Rad Laboratories, Inc.) according to the manufacturer's protocol. Protein bands were visualized and the detection of band gray value was performed using Image Studio software (version 4.0; LI-COR Biosciences). $\beta$-actin and GAPDH were used as the loading controls.

Immunohistochemistry (IHC) staining. The tumor samples were collected and fixed in 4\% paraformaldehyde for $24-48 \mathrm{~h}$, then embedded in paraffin. Paraffin-embedded tissue samples were serially cut (4- $\mu \mathrm{m}$ thick) and dried overnight at $60^{\circ} \mathrm{C}$. The samples were then deparaffinized in xylene at room temperature and rehydrated using a graded descending series of ethanol (100, 95 and 80\%). Antigen retrieval was performed in boiling water using $10 \mathrm{mM}$ citrate solution for $5 \mathrm{~min}$, and endogenous hydrogen peroxidase activity was blocked by incubation with $10 \%$ hydrogen peroxide for $30 \mathrm{~min}$ at room temperature. Each of these aforementioned steps was followed by a 10 min PBS wash. Sections were blocked with $3 \% \mathrm{H}_{2} \mathrm{O}_{2}$ (cat. no. SP-9002; OriGene Technologies, Inc.) at $37^{\circ} \mathrm{C}$ for $30 \mathrm{~min}$. The samples were subsequently incubated overnight with an anti-SMARCC2 (1:50; cat. no. ab243634; Abcam) primary antibody at $4^{\circ} \mathrm{C}$. Following the primary antibody incubation, the sections were incubated with a goat anti-rabbit secondary antibody (1:1,000; cat. no. SP-9002; OriGene Technologies, Inc.) at room temperature for $2 \mathrm{~h}$. The sections were then stained with diaminobenzidine at room temperature for 3-5 min and counterstained with hematoxylin at room temperature for $5 \mathrm{~min}$. Finally, images were captured using an Olympus light microscope (Olympus Corporation) under x200 magnification. Positive staining of SMARCC2 was scored from 0-3 according to the intensity of the staining: 0 (negative), 1 (weakly positive, light yellow), 2 (moderately positive, yellowish brown) and 3 (strongly positive, brown). The percentage of positively-stained cells was also scored using the following scores: $0(0 \%), 1(1-33 \%), 2(34-66 \%)$ and $3(67-100 \%)$. The sum of the intensity and percentage scores was used as the final staining score.
Co-immunoprecipitation (Co-IP) assay. The cells were harvested and the total protein was extracted using lysis buffer for western and IP assays (cat. no. 6505706) with protease-phosphatase inhibitor (EMD Millipore). The protein lysate was centrifuged at $14,000 \mathrm{x} \mathrm{g}$ at $4^{\circ} \mathrm{C}$ for $15 \mathrm{~min}$ and the supernatant was collected. U87MG and LN229 cells were seeded into $10-\mathrm{cm}$ dishes at a density of $5 \times 10^{5}$ cells/dish and incubated for $24 \mathrm{~h}$. Equal amounts of protein were coimmunoprecipitated with a rabbit anti-SMARCC2 (1:1,000; cat. no. ab243634; Abcam), rat anti-c-Myc (1:1,000; cat. no. ab32072; Abcam), anti-tumor protein D52 (TPD52) like 2 (TPD52L2; 1:1,000; cat. no. ab234819; Abcam) monoclonal antibody at $4^{\circ} \mathrm{C}$ overnight and then incubated with protein $\mathrm{A} / \mathrm{G}(1: 1)$ sepharose magnetic beads $(40 \mu \mathrm{l})\left(\mathrm{GE}\right.$ Healthcare) at $4^{\circ} \mathrm{C}$. The total volume was $400 \mu 1$. Following incubation, lysis buffer was used for washing. After magnetic separation, the supernatants with unbound free proteins were separated and the protein complex was resuspended in SDS buffer for western blotting analyses, which were performed according to the aforementioned protocols. A rabbit anti-IgG antibody (GeneTex, Inc.) was used as the negative control.

Wound healing assay. Cell migration was determined using a wound healing assay. Briefly, U87MG/T98G/LN229 cells were cultured to $100 \%$ confluence in a six-well plate and then the cell monolayer was scratched with a 1-ml pipette tip to create an artificial wound. The detached cells were removed with PBS and remaining cells were cultured in high-sugar serum-free DMEM. Images of the same wound area were photographed at 0 , and 24 or $48 \mathrm{~h}$ under a light microscope (magnification, $\mathrm{x} 40$ ). The healed scratch area was calculated using Image J 1.8.0 software (National Institutes of Health) using the following formula: Healed scratch $(\%)=[($ initial scratch area-final scratch area)/initial scratch area] x100.

Cell invasion and migration assays. Cell invasion and migration was investigated using Transwell plates (Costar; Corning, Inc.) with a pore size of $0.8-\mu \mathrm{m}$. Briefly, $5 \times 10^{5}$ U87MG/T98G/LN229 cells were seeded in high-sugar serum-free DMEM into the upper chamber of the Transwell plates, which was precoated with Matrigel at $37^{\circ} \mathrm{C}$ overnight (for the invasion assay). Medium supplemented with 10\% FBS was plated into the lower chamber. Following incubation for 6-8 h at $37{ }^{\circ} \mathrm{C}$, non-migratory or non-invasive cells in the upper chamber were removed, whereas migratory and invasive cells in the lower chamber were fixed with $20 \%$ methanol for $5 \mathrm{~min}$ and stained for $5 \mathrm{~min}$ with $0.1 \%$ crystal violet. The cells from six randomly selected fields of view were observed under a confocal microscope (Olympus CX23; Olympus Corporation) at x200 magnification, then ImageJ software (version 1.8.0; National Institutes of Health) was used to calculate the number of invasive and migrated cells.

Bioinformatics analysis. Level 3 RNA-SeqV2 data (containing data on genes, isoforms, exons and junction levels), level 3 Agilent microarray gene expression data and clinical data for LGG and GBM were downloaded from The Cancer Genome Atlas (TCGA) database (cancer.gov/about-nci/organization/ ccg/research/structural-genomics/tcga) using the TCGA biolinks package cgdsr (github.com/cBioPortal/cgdsr) on $\mathrm{R}$ 
platform (version 3.6.3). Data were obtained from the TCGA data sets 'lgg_tcga' and 'gbm_tcga'. The cut-off mode based on median SMARCC2 expression was selected without specifying a track subset.

Statistical analysis. Statistical analyses were performed using SPSS 20.0 software (IBM Corp.). All experiments were performed in triplicate and data are presented as the mean \pm SEM.Comparisons between two groups were performed using paired Student's t-test or a Mann-Whitney U test, whereas statistical differences among several groups were determined using one-way ANOVA followed by Dunnett's post hoc test or the Kruskal Wallis test followed by Dunn's post hoc test. Survival plots were generated using the Kaplan-Meier method and log-rank test; in instances where there was late-stage crossover between the groups, Cramer-von Mises tests were used. $\mathrm{P}<0.05$ was considered to indicate a statistically significant difference.

\section{Results}

Expression levels of SMARCC2 in patients with glioma with different tumor grades. The results of the bioinformatics analyses revealed that SMARCC2 was differentially expressed in different grades of glioma (Fig. 1A); as glioma grade increased, expression levels of SMARCC2 decreased and high expression levels of SMARCC2 were significantly associated with an improved prognosis in patients with glioma (Fig. 1B). Furthermore, overall survival time was separately analyzed in low-grade gliomas (LGG) and high-grade gliomas (GBM), and the results revealed no significant difference in overall survival time between GBMs with high or low SMARCC2 expression (Fig. S1). LGG shows that high expression of SMARCC2 has a better prognosis. (Fig. S1). An SMARCC2-specific antibody was used for the IHC analysis of different glioma grades (Fig. 1C), and a standardized scoring method was used to analyze the results of the IHC analysis. In the WHO III and IV group, the protein expression levels of SMARCC2 were significantly downregulated compared with the low-grade group (Fig. 1D). RT-qPCR analysis revealed that the mRNA expression levels of SMARCC2 were also significantly downregulated in tissues (WHO I-IV; Fig. 1E), which was consistent with the results for SMARCC2 protein expression levels. These decreases were positively associated with severity of the tumor.

Effects of SMARCC2 knockdown on glioma cell migration and invasion. siRNAs targeting SMARCC2 were transfected into human glioma cancer cells, U87MG and T98G, and the protein and mRNA expression levels of SMARCC2 were analyzed via western blotting (Fig. 2A) and RT-qPCR (Fig. 2B), respectively, after $48 \mathrm{~h}$. The expression levels of SMARCC2 were downregulated in the siSMARCC2-1 and siSMARCC2-3 groups compared with the NC group at both the protein and mRNA levels. Subsequently, Transwell assays were performed to determine the effect of SMARCC2 on glioma cell migration and invasion; the results revealed that the knockdown of SMARCC2 expression significantly increased the number of migratory and invasive cells compared with the NC group (Fig. 2C). Furthermore, wound healing assays were performed to further validate the effect of SMARCC2 on cell migration (Fig. 2D). Compared with the NC group, GBM cell migration was significantly increased in the siSMARCC2-1 and siSMARCC2-3 groups.

Overexpression of SMARCC2 inhibits glioma cell migration and invasion. To determine the potential biological functions of SMARCC2 in glioma cell migration and invasion, U87MG and LN229 cell lines stably overexpressing SMARCC2 were established by infecting cells with recombinant adenovirus vectors. The transduction efficiency was analyzed by the intensity of GFP fluorescence at $48 \mathrm{~h}$ post-transduction (data not shown). The transduction efficiency was determined as $90-95 \%$. The protein and mRNA expression levels of SMARCC2 in U87MG and LN229 cancer cells following the overexpression of SMARCC2 were also analyzed via western blotting (Fig. 3A) and RT-qPCR (Fig. 3B), respectively. Following transfection and overexpression of lentivirus, the RNA and protein levels of SMARCC2 increased significantly. Transwell (Fig. 3C) and wound healing assays (Fig. 3D) were conducted to analyze cell migration and invasion following the overexpression of SMARCC2. The results demonstrated that the transduction of cells with SMARCC2 (oe) adenovirus significantly decreased U87MG and LN229 cell migration and invasion compared with the LVS-NC group.

SMARCC2 directly targets $c-M y c$ and inhibits its oncogenic functions. Co-IP experiments were performed to analyze the interactions between SMARCC2 and c-Myc (Fig. 4A and B). SMARCC2 was found to interact with c-Myc. Notably, SMARCC2 also strongly interacted with TPD52L2 (Fig. 4C and D), which is a member of the TPD52 family (21). Furthermore, the western blotting results indicated that the overexpression of SMARCC2 notably downregulated the expression levels of c-Myc compared with the LVS-NC group (Fig. 4E and F). These findings suggested that SMARCC2 may inhibit the oncogenic function of c-Myc by promoting c-Myc protein degradation.

SMARCC2 induces EMT in glioma cells by targeting the Wnt/ $\beta$-catenin signaling pathway. EMT is a key process through which epithelial cells acquire mesenchymal properties, and it has been closely associated with cancer invasion and metastasis (22). The expression levels of a number of EMT markers were analyzed in U87MG and LN229 cells overexpressing SMARCC2. The present results revealed that the expression levels of T-cadherin were markedly upregulated, whereas the expression levels of $\mathrm{N}$-cadherin, $\beta$-catenin, vimentin and Snail were notably downregulated in cells transfected with SMARCC2 (oe) adenovirus compared with LVS-NC (Fig. 5A and B). Subsequently, a Wnt/ $\beta$-catenin signaling pathway agonist, HLY78, was used for rescue experiments. The results demonstrated that overexpression of SMARCC2 in combination with HLY78 treatment markedly reversed the agonistic effect of HLY78 on the Wnt/ $\beta$-catenin signaling pathway (Fig. 5C and D). The expression of EMT-associated proteins $(\mathrm{N}$-cadherin/snail/vimentin/ $\beta$-catenin) increased significantly following treatment with HLY78-alone, but decreased when SMARCC2 was simultaneously overexpressed and treated with HLY78. 
A

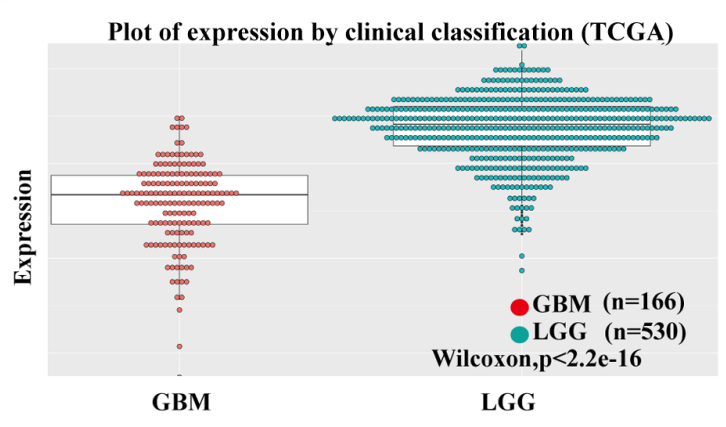

C

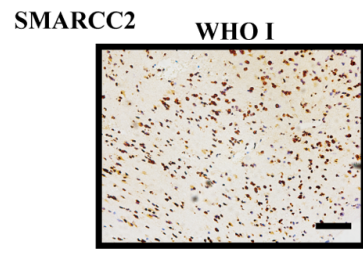

WHO III

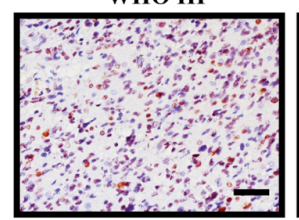

$\mathbf{E}$

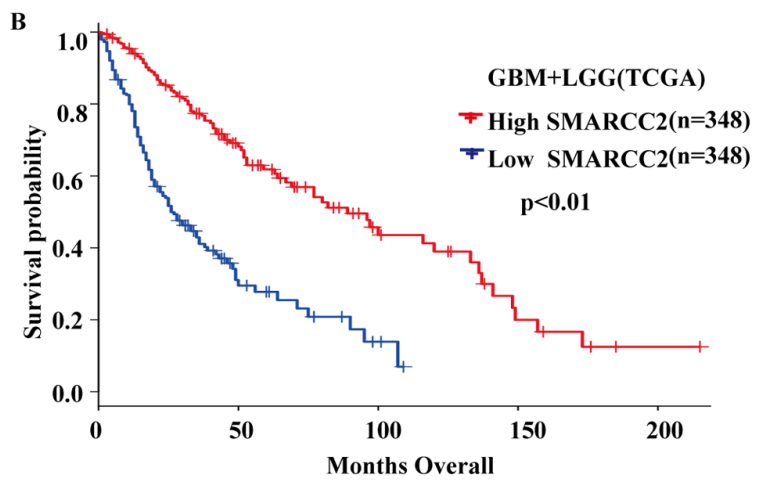

D

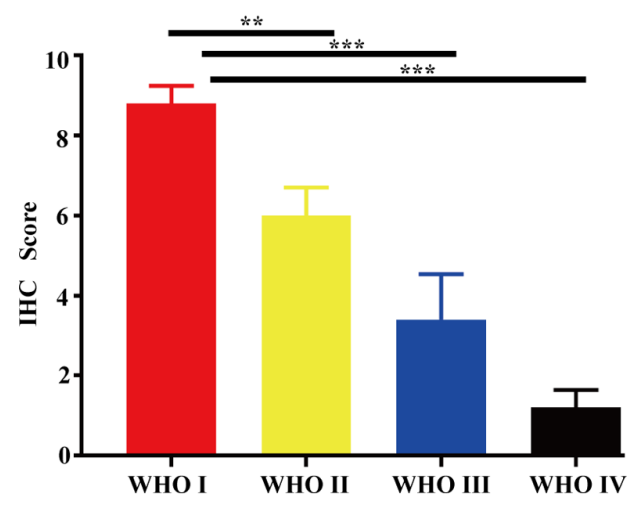

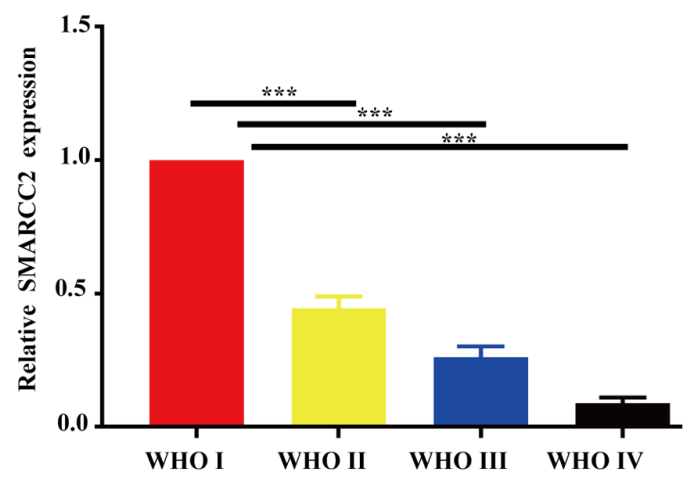

Figure 1. SMARCC2 expression and survival analysis in patients with different grades of glioma. (A) Expression levels of SMARCC2 differed in patients with LGG and GBM obtained from TCGA. (B) Survival analysis indicated that patients with high SMARCC2 expression displayed an improved prognosis compared with patients with low expression. (C) IHC staining of tumors from patients with different glioma grades revealed a negative association between SMARCC2 expression and glioma grade. (D) Statistical analysis of IHC staining scores. (E) Reverse transcription-quantitative PCR analysis revealed that the expression levels of SMARCC2 were upregulated in low-grade glioma and decreased as the disease severity increased. ${ }^{* *} \mathrm{P}<0.05 ;{ }^{* * *} \mathrm{P}<0.01$. SMARCC2, SWItch/sucrose non-fermentable related, matrix associated, actin dependent regulator of chromatin subfamily c member 2; TCGA, The Cancer Genome Atlas; WHO, World Health Organization; GBM, glioblastoma; LGG, low-grade glioma; IHC, immunohistochemistry.

\section{Discussion}

The molecular structure of the ATP-dependent chromatin-remodeling complex and its effect on the growth and development of individuals have been extensively studied (23-25). Chromatin remodelers are specialized multi-protein machines that enable access to nucleosomal DNA by altering the structure, composition and positioning of nucleosomes (26). Mutations in the SWI/SNF family of proteins have been shown to be involved in the development of a number of cancer cell types (including breast, colon, liver and stomach cancer); however, to the best of our knowledge, the underlying mechanism remains unknown (27-29). The present study aimed to determine whether the core subunit of SWI/SNFs, SMARCC2, served a tumor suppressive or oncogenic role in glioma cells.

The expression of SMARCC2 is associated with glioma grade. High-grade gliomas show lower expression of SMARCC2, and the higher the expression of SMARCC2, the better the prognosis. SMARCC2 may have a tumor suppressor function. When the interference rna fragment was used to knock down expression of SMARCC2, GBM showed stronger migration and invasion capabilities. When oe lentiivirus was used to increase expression of SMARCC2, the migration and invasion capabilities of GBM and expression levels of EMT-associated proteins (N-cadherin/snail/vimentin/ $\beta$-catenin) significantly decreased. 

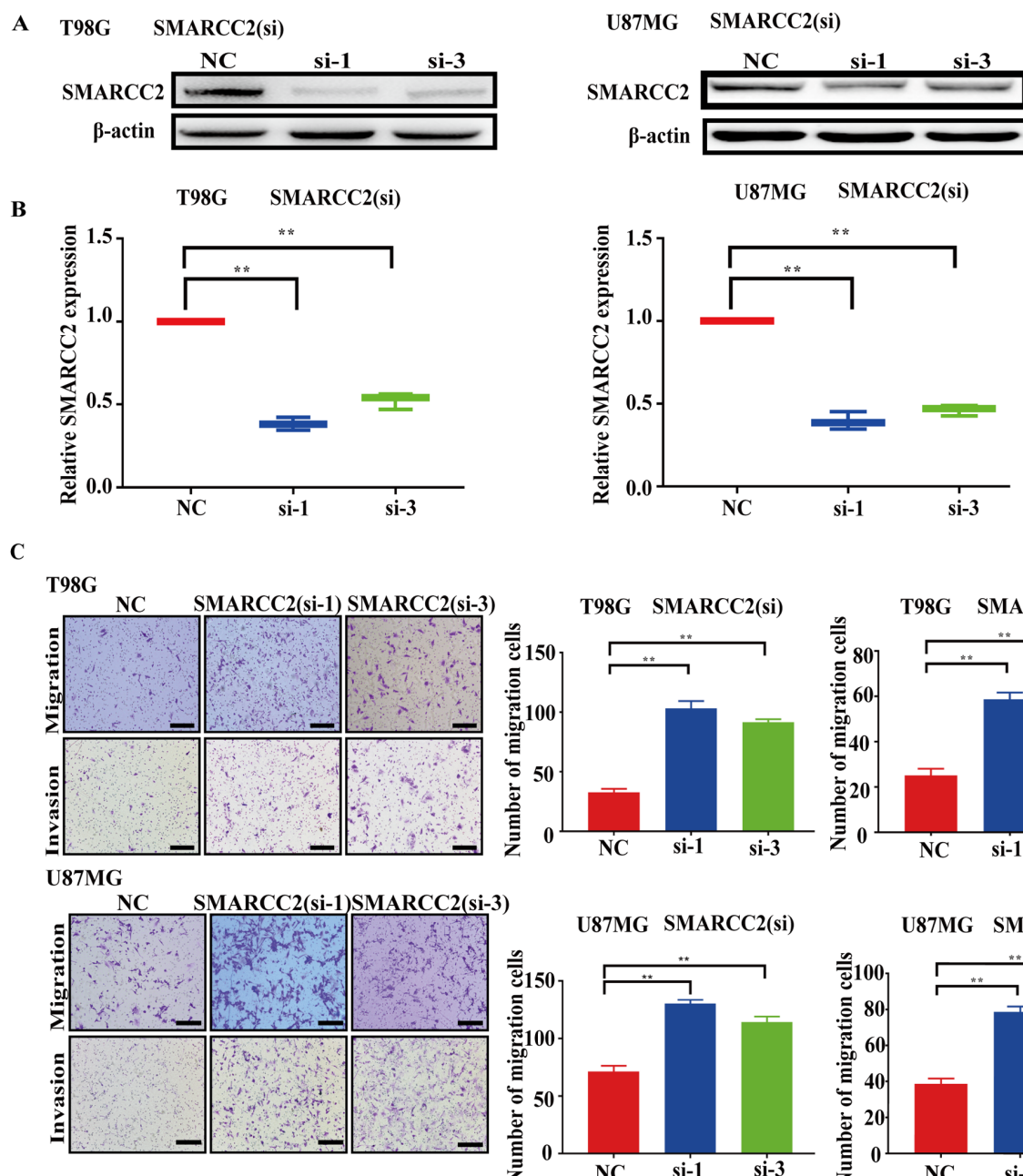

C
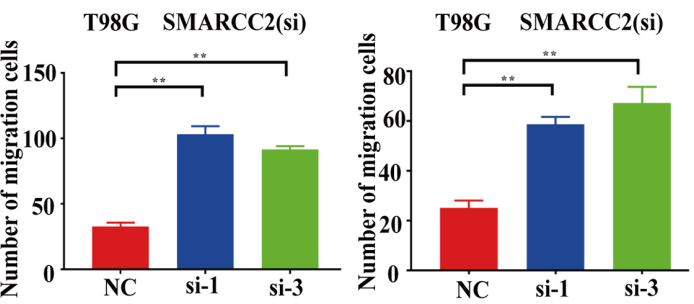

U87MG SMARCC2(si)

U87MG SMARCC2(si)
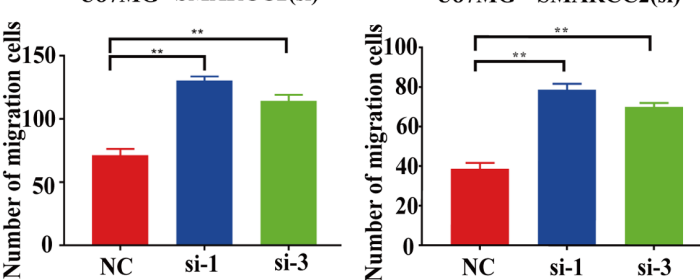

D
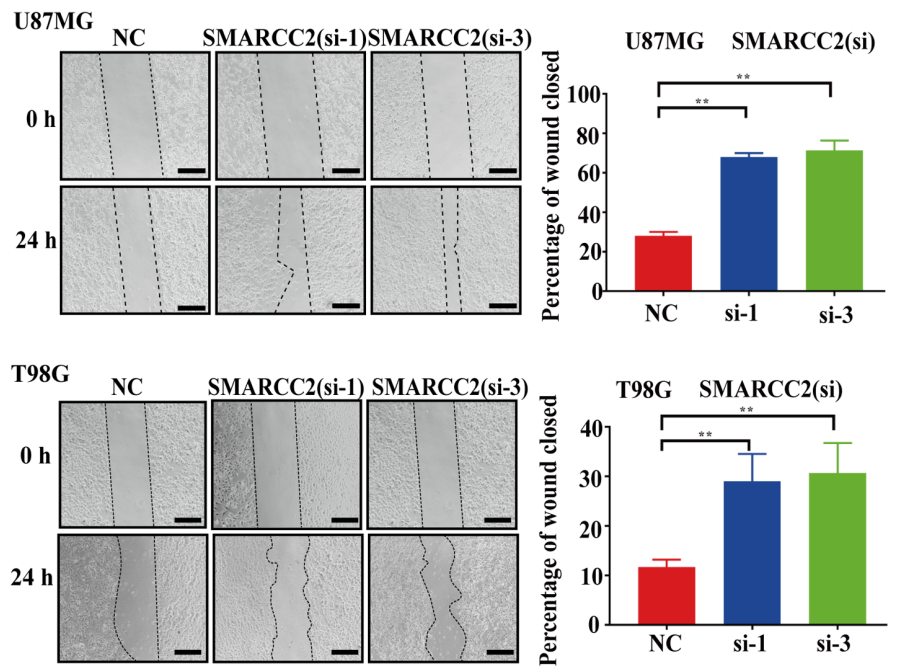

Figure 2. SMARCC2 knockdown enhances the migration and invasion ability of T98G and U87MG cells. (A) Analysis of SMARCC2 expression levels in T98G and U87MG cells transfected with siSMARCC2 was performed via western blotting. $\beta$-actin was used as the internal control. (B) mRNA expression levels of SMARCC2 in T98G and U87MG cells were analyzed via reverse transcription-quantitative PCR. GAPDH was used as the internal control. (C) Representative images and quantification of the Transwell assays showing the different migratory and invasive abilities of NC- and siSMARCC2-1/3-transfected cells. Scale bar, $100 \mathrm{~mm}$. (D) Representative images and quantification of the wound healing assay following transfection of siSMARCC2-1/3 into U87MG and T98G cells Scale bar, $200 \mathrm{~mm} .{ }^{* *} \mathrm{P}<0.05$. SMARCC2, SWItch/sucrose non-fermentable related, matrix associated, actin dependent regulator of chromatin subfamily c member 2; si, small interfering RNA; NC, negative control.

The results of the present study suggested that SMARCC2 may serve as a tumor suppressor gene in glioma, inhibiting glioma cell migration and invasion. Moreover, SMARCC2 was shown to interact with c-Myc, which reportedly serves a role in the $\mathrm{Wnt} / \beta$-catenin signaling pathway (30-33). SMARCC2 inhibited the EMT process of GBM cell 
$\mathbf{A}$

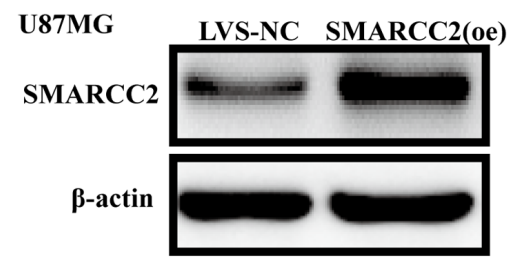

B

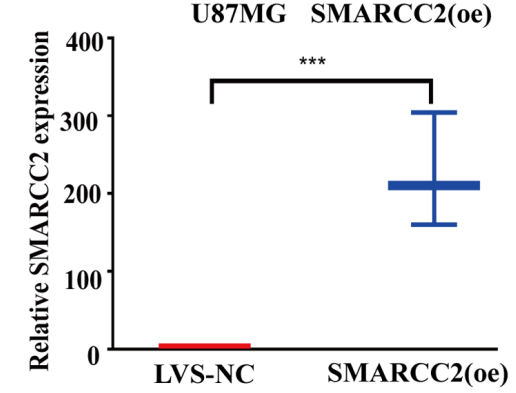

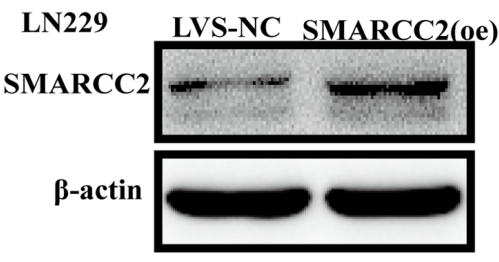

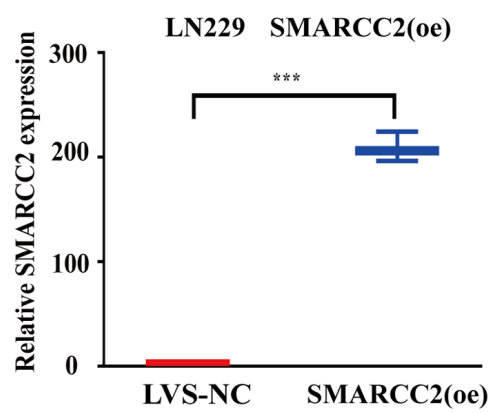

C
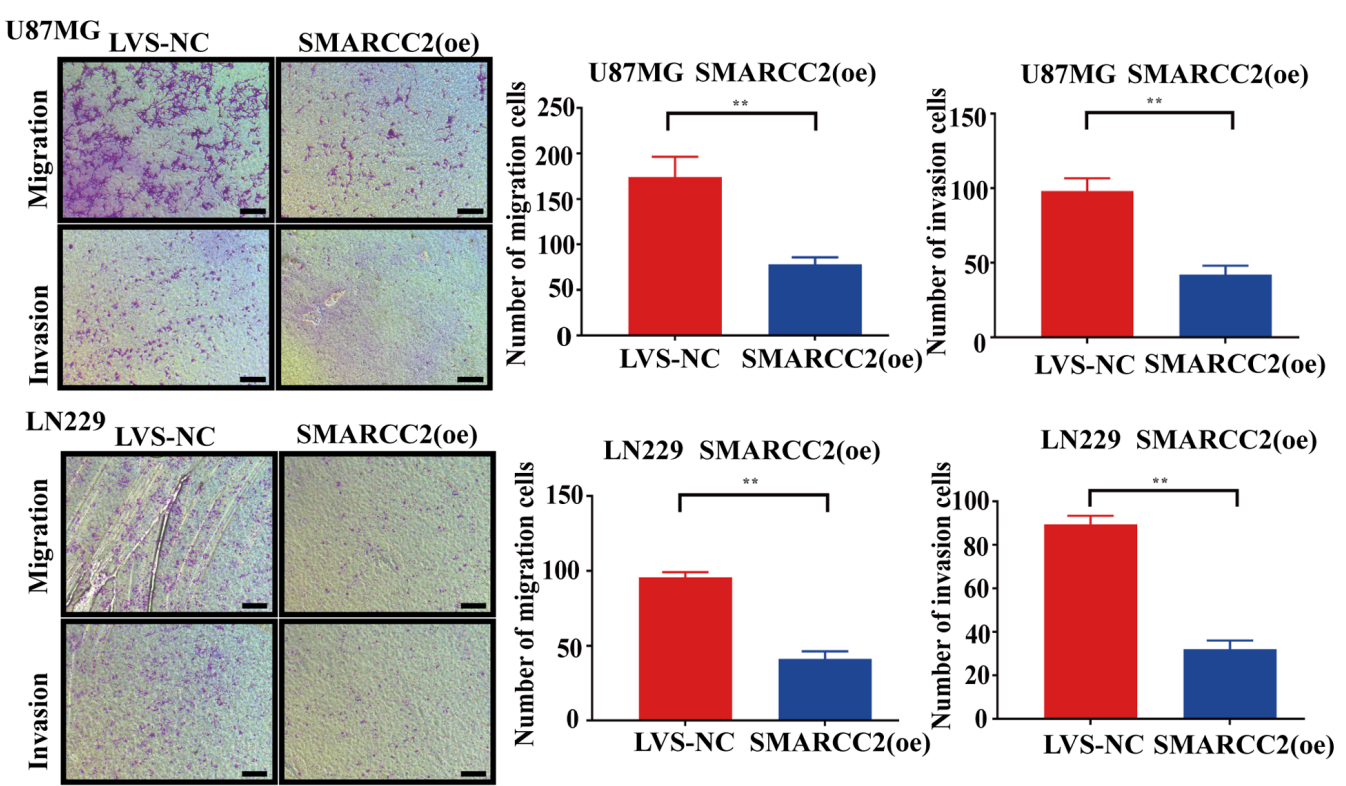

D
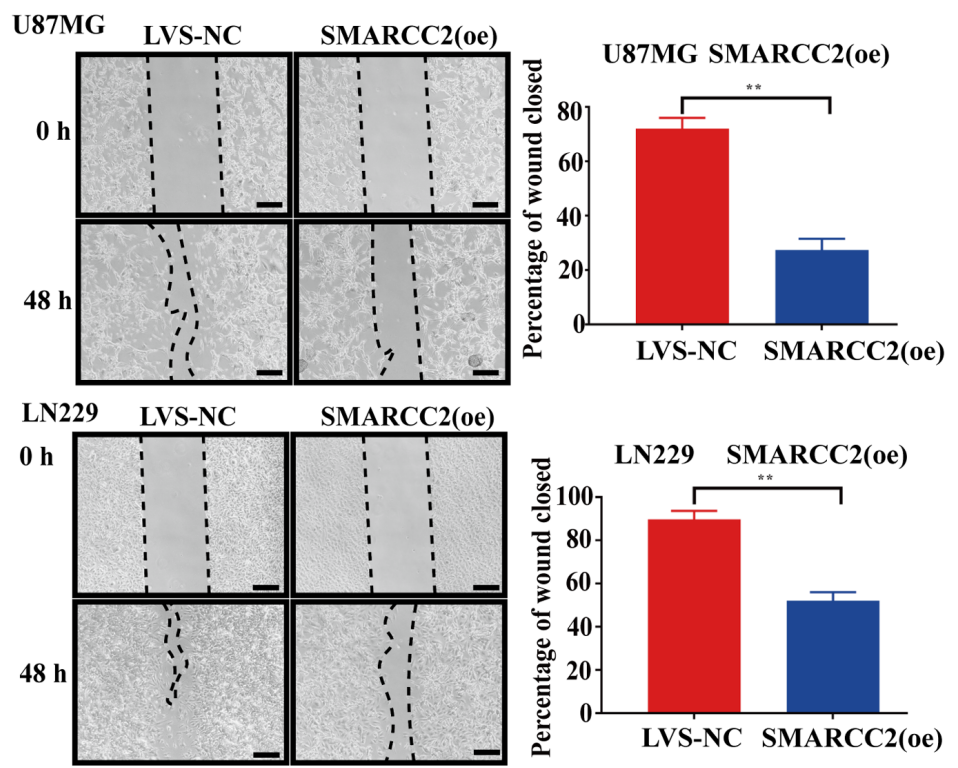

Figure 3. SMARCC2 overexpression attenuates the migratory and invasive abilities of U87MG and LN229 cells. Transduction efficiency of SMARCC2 (oe) recombinant adenovirus in U87MG and LN229 cell lines was analyzed via (A) western blotting and (B) reverse transcription-quantitative PCR. (C) Representative images and quantification of the Transwell assays showing the different migratory and invasive abilities of LVS-NC- and SMARCC2 (oe)-transfected cells. Scale bar, $100 \mathrm{~mm}$. (D) Representative images and quantification of the wound healing assay following transduction with SMARCC2 (oe) adenovirus in U87MG and LN229 cells. Scale bar, $200 \mathrm{~mm} .{ }^{* *} \mathrm{P}<0.05 ;{ }^{* * * *} \mathrm{P}<0.01$. SMARCC2, SWItch/sucrose non-fermentable related, matrix associated, actin dependent regulator of chromatin subfamily c member 2; LVS-NC, lentiviral vector-non-specific control; NC, negative control; oe, overexpression. 
A U87MG IP-SMARCC2

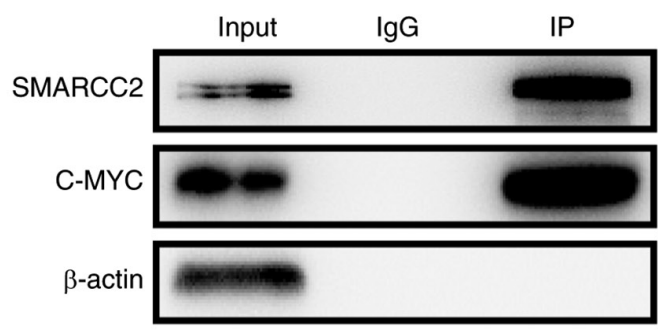

C U87MG

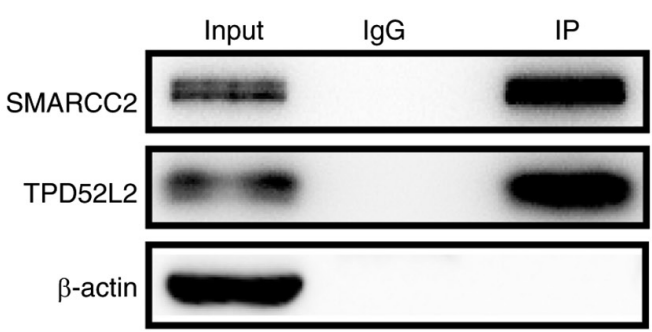

E U87MG

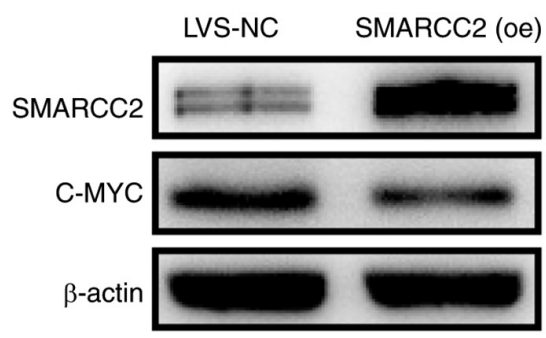

B LN229

\section{IP-SMARCC2}

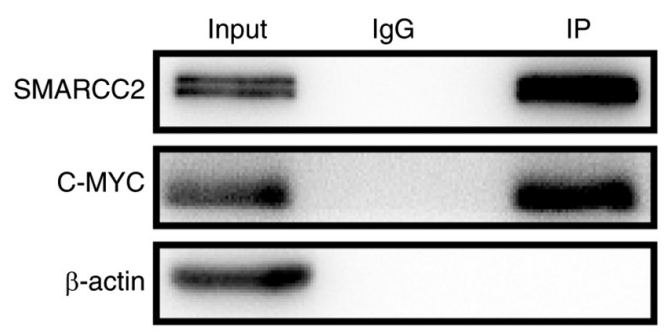

LN229

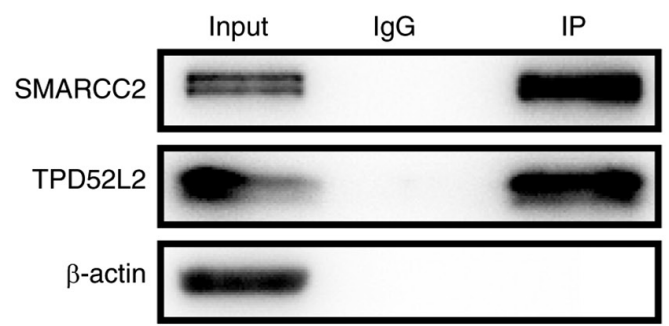

$F_{\text {LN229 }}$

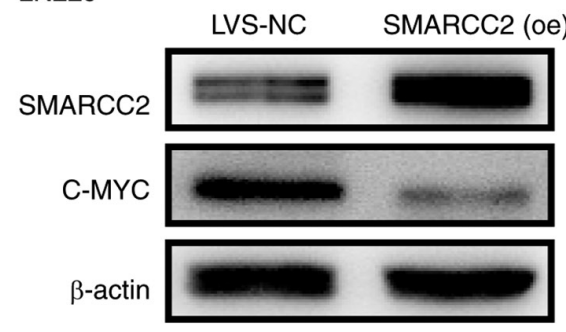

Figure 4. Identification of proteins that interact with SMARCC2 by performing Co-IP experiments. An interaction between SMARCC2 and c-Myc was detected in (A) U87MG and (B) LN229 cell lines. An interaction between SMARCC2 and TPD52L2 was detected in (C) U87MG and (D) LN229 cell lines. Analysis of c-Myc protein expression levels in (E) U87MG and (F) LN229 cells overexpressing SMARCC2. SMARCC2, SWItch/sucrose non-fermentable related, matrix associated, actin dependent regulator of chromatin subfamily c member 2; IP, immunoprecipitation; oe, overexpression; LVS, lentivirus; $\mathrm{NC}$, negative control; TPD52L2, tumor protein D52 like 2.

lines by downregulating T-cadherin and upregulating $\mathrm{N}$-cadherin/ $\beta$-catenin/snail/vimentin. SMARCC2 was also discovered to regulate EMT via the Wnt/ $\beta$-catenin signaling axis; therefore, it was hypothesized that c-Myc may participate in SMARCC2-mediated regulation of EMT. These results were consistent with the results of previous studies investigating SWI/SNF mutations in a number of types of cancer (34-36). The present results showed differential expression of SMARCC2 in glioma of different grades.

The SWI/SNF complex has been demonstrated to exert essential roles in the life cycle of cells and was found to participate in the developmental maturation of various types of neural cell $(37,38)$. As the core subunit of SWI/SNF, SMARCC2 may be responsible for these functions in neural cells, thus indicating its role as a possible tumor suppressor gene. In the absence of SMARCC2, cells cannot develop into mature functional neuronal cells. The present study transfected two GBM cell lines (U87MG and LN229) with SMARCC2 (oe) adenoviruses, which resulted in significantly increased expression levels of SMARCC2 compared with the LVS-NC group, as demonstrated via RT-qPCR and western blotting. In U87MG and LN229 cells, the expression of SMARCC2 was significantly lower than in T98G cells. It was hypothesized that there is a SMARCC2 gene mutation in the U87MG and LN229 cell line, resulting in low expression of SMARCC2. These results also validated that there were mutations in the U87MG and LN229 cell lines.

The results of the present study indicated that SMARCC2 may interact with c-Myc to inhibit its oncogenic functions in U87MG and LN229 cells. Nevertheless, the underlying mechanism of action remains unclear. TPD52L2 is a member of the TPD52 family that has been implicated in multiple types of human cancer (39-41). Further research has demonstrated that the TPD52 gene encodes regulators of cancer cell proliferation, indicating that TPD52 may be important for maintaining tumorigenesis and metastasis of cancer cells (42). Our previous study found that TPD52L2 is associated with the EMT process of the GBM cell line; the present study demonstrated interaction between SMARCC2 and TPD52L2, further suggesting that SMARCC2 affects the EMT process of the GBM cell line. However, one possible mechanism is that SMARCC2 may serve as a post-translationally modified protease to promote c-Myc protein degradation, which will be the focus of future research.

In conclusion, the findings of the present study suggested that SMARCC2, as the core subunit of SWI/SNF, may be involved 
A U87MG

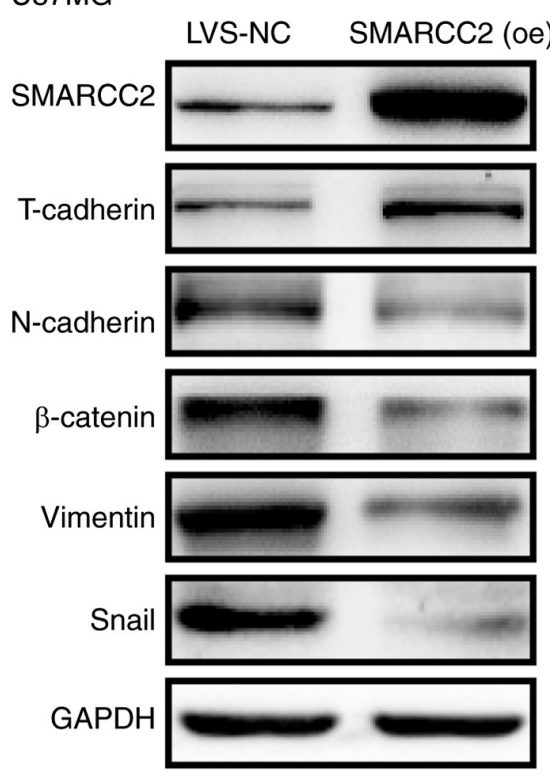

C U87MG

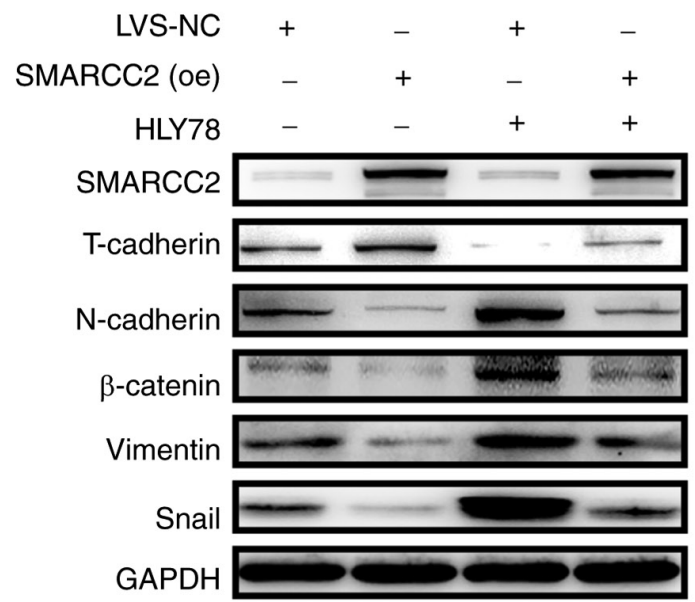

B $\quad$ LM229

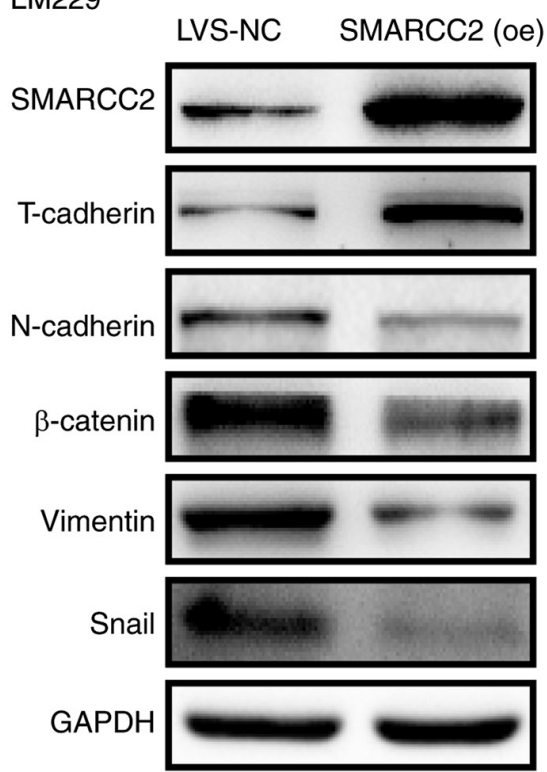

D LM229

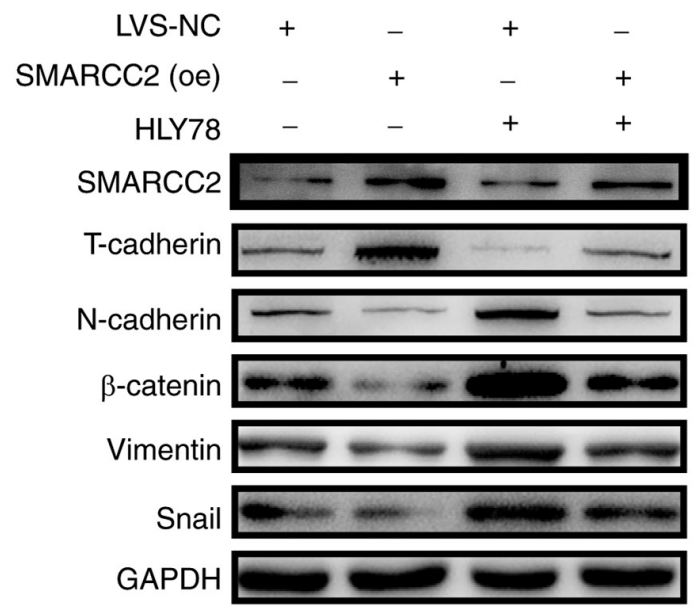

Figure 5. Analysis of the expression levels of EMT-related proteins in cells overexpressing SMARCC2.Expression levels of EMT-associated markers T-cadherin, $\mathrm{N}$-cadherin, $\beta$-catenin, vimentin and Snail were analyzed via western blotting in (A) U87MG and (B) LM229 cells overexpressing SMARCC2. GAPDH was used as the internal control. Western blotting was performed to determine the expression levels of EMT-related proteins in (C) U87MG and (D) LM229 cells following overexpression of SMARCC2 and treatment with the Wnt/B-catenin signaling pathway agonist, HLY78. EMT, epithelial-mesenchymal transition; SMARCC2, SWItch/sucrose non-fermentable related, matrix associated, actin dependent regulator of chromatin subfamily c member 2; oe, overexpression; LVS-NC, lentiviral vector-non-specific control; NC, negative control; Snail, snail family transcriptional repressor 1.

in the occurrence, development and prognosis of GBM.. These results may provide novel insight into the molecular mechanisms underlying glioma formation and progression, and may provide a new potential target for molecular-targeted therapies and prognostic markers for glioma.

\section{Acknowledgements}

Not applicable.

\section{Funding}

The present study was supported by the National Natural Science Foundation of China (grant no. 81802830).

\section{Availability of data and materials}

The datasets used and/or analyzed during the current study are available from the corresponding author on reasonable request.

\section{Authors' contributions}

CL, YL and QZ made substantial contributions to the study conception. CL designed the study. CF performed the experiments and analyzed and interpreted the data. HW and RR drafted and critically revised the manuscript for important intellectual content. RR made substantial contributions to data analysis. JL, HW, LC, HL, LS, CS and JG made substantial contributions to 
acquisition of data. CL and CF confirm the authenticity of all the raw data. All authors read and approved the final manuscript.

\section{Ethics approval and consent to participate}

The present study protocol was approved by the Institutional Review Board at Nanfang Hospital of Southern Medical University. All research was performed in accordance with the principles of the Declaration of Helsinki of 1975. All patients provided written informed prior to participation in the study, and all study results were stored and analyzed anonymously.

\section{Patient consent for publication}

Not applicable.

\section{Competing interests}

The authors declare that they have no competing interests.

\section{References}

1. Wilson BG and Roberts CW: SWI/SNF nucleosome remodellers and cancer. Nat Rev Cancer 11: 481-492, 2011.

2. He S, Wu Z, Tian Y, Yu Z, Yu J, Wang X, Li J, Liu B and $\mathrm{Xu}$ Y: Structure of nucleosome-bound human BAF complex. Science 367: 875-881, 2020.

3. Zhou Y, Johnson SL, Gamarra NI and Narlikar GJ: Mechanisms of ATP dependent chromatin remodeling motors. Annu Rev Biophys 45: 153-181, 2016.

4. Clapier R, Iwasa J, Cairns BR and Peterson CL: Mechanisms of action and regulation of ATP-dependent chromatin-remodelling complexes. Nat Rev Mol Cell Biol 18: 407-422, 2017.

5. Saha A, Wittmeyer J and Cairns BR: Chromatin remodelling: The industrial revolution of DNA around histones. Nat Rev Mol Cell Biol 7: 437-447, 2006.

6. Kassabov SR, Zhang B, Persinger J and Bartholomew B: SWI/SNF unwraps, slides, and rewraps the nucleosome. Mol Cell 11: 391-403, 2003.

7. Sati S and Cavalli G: Chromosome conformation capture technologies and their impact in understanding genome function. Chromosoma 126: 33-44, 2017.

8. Masliah-Planchon J, Bièche I, Guinebretière JM, Bourdeaut F and Delattre O: SWI/SNF chromatin remodeling and human malignancies. Ann Rev Pathol 10: 145-171, 2015.

9. Kadoch C, Hargreaves DC, Hodges C, Elias L, Ho L, Ranish J and Crabtree GR: Proteomic and bioinformatic analysis of mammalian SWI/SNF complexes identifies extensive roles in human malignancy. Nat Genet 45: 592-601, 2013.

10. Almeida R, Fernández-Justel JM, Santa-María C, Cadoret JC, Cano-Aroca L, Lombraña R, Herranz G, Agresti A and Gómez M: Chromatin conformation regulates the coordination between DNA replication and transcription. Nat Commun 9: 1590, 2018.

11. Qiang Z, Jun-Jie L, Hai W, Hong L, Bing-Xi L, Lei C, Wei X, Ya-Wei L, Huang A, Song-Tao Q and Yun-Tao L: TPD52L2 impacts proliferation, invasiveness and apoptosis of glioblastoma cells via modulation of $\mathrm{wnt} / \beta$-catenin/snail signaling. Carcinogenesis 39: 214-224, 2018.

12. Li H, Chen L, Qi S, Yu S, Weng Z, Hu Z, Zhou Q, Xin Z, Shi L, Ma L, et al: HERC3-mediated SMAD7 ubiquitination degradation promotes autophagy-induced EMT and chemoresistance in glioblastoma. Clin Cancer Res 25: 3602-3616, 2019.

13. Jackson CM, Choi J and Lim M: Mechanisms of immunotherapy Resistance: Lessons from glioblastoma. Nat Immunol 20 1100-1109, 2019.

14. Tomiyama A and Ichimura K: Signal transduction pathways and resistance to Targeted therapies in glioma. Semin Cancer Biol 58: 118-129, 2019.

15. Tan MS, Sandanaraj E, Chong YK, Lim SW, Koh LW, Ng WH, Tan NS, Tan P, Ang BT and Tang C: A STAT3-based gene signature stratifies glioma patients for targeted therapy. Nat Commun 10: 3601, 2019 .
16. Huang K, Liu X, Li Y, Wang Q, Zhou J, Wang Y, Dong F, Yang C, Sun Z, Fang C, et al: Genome-Wide CRISPR-Cas9 screening identifies $\mathrm{NF}-\kappa \mathrm{B} / \mathrm{E} 2 \mathrm{~F} 6$ responsible for EGFRvIII-associated temozolomide resistance in glioblastoma. Adv Sci (Weinh) 6: $1900782,2019$.

17. Annibali D, Whitfield JR, Favuzzi E, Jauset T, Serrano E, Cuartas I, Redondo-Campos S, Folch G, Gonzàlez-Juncà A, Sodir NM, et al: Myc inhibition is effective against glioma and reveals a role for Myc in profificient mitosis. Nat Commun 5: 4632, 2014.

18. Sang Y, Li Y, Zhang Y, Alvarez A A, Yu B, Zhang W, Hu B, Cheng SY and Feng H: CDK5-dependent phosphorylation and nuclear Translocation of TRIM59 promotes macroH2A1 ubiquitination and tumorigenicity. Nat Commun 10: 4013, 2019.

19. Weller M, Wick W, Aldape K, Brada M, Berger M, Pfister SM, Nishikawa R, Rosenthal M, Wen PY, Stupp R and Reifenberger G: Glioma. Nat Rev Dis Primers 1: 15017, 2015.

20. Livak KJ and Schmittgen TD: Analysis of relative gene expression data using real-time quantitative PCR and the 2(-Delta Delta C(T)) method. Method 25: 402-408, 2001.

21. Yang M, Wang X, Jia J, Gao H, Chen P, Sha X and Wu S: Tumor protein D52-like 2 contributes to proliferation of breast cancer cells. Cancer Biother Radiopharm 30: 1-7, 2014.

22. Zhang J, Cai H, Sun L, Zhan P, Chen M, Zhang F, Ran Y and Wan J: LGR5, a novel functional glioma stem cell marker, promotes EMT by activating the $\mathrm{Wnt} / \beta$-catenin pathway and predicts poor survival of glioma patients. J Exp Clin Cancer Res 37: 225, 2018.

23. Savas S and Skardasi G: The SWI/SNF complex subunit genes: Their functions, variations, and links to risk and survival outcomes in human cancers. Crit Rev Oncol Hematol 123: 114-131, 2018.

24. Bögershausen N and Wollnik B: Mutational landscapes and phenotypic spectrum of SWI/SNF-related intellectual disability disorders. Front Mol Neurosci 11: 252, 2018.

25. Alver BH, Kim KH, Lu P, Wang X, Manchester HE, Wang W, Haswell JR, Park PJ and Roberts CW: The SWI/SNF chromatin remodelling complex is required for maintenance of lineage specific enhancers. Nat Commun 8: 14648, 2017.

26. Pillidge $Z$ an Bray SJ: SWI/SNF chromatin remodeling controls Notch-responsive enhancer accessibility. EMBO Rep 20: e46944, 2019.

27. Wang W, Friedland SC, Guo B, O'Dell MR, Alexander WB, Whitney-Miller CL, Agostini-Vulaj D, Huber AR, Myers JR, Ashton JM, et al: ARID1A, a SWI/SNF subunit, is critical to acinar cell homeostasis and regeneration and is a barrier to transformation and epithelial-mesenchymal transition in the pancreas. Gut 68: 1245-1258, 2019.

28. Wang $X$, Lee RS, Alver BH, Haswell JR, Wang S, Mieczkowski J, Drier Y, Gillespie SM, Archer TC, Wu JN, et al: SMARCB1-mediated SWI/SNF complex function is essential for enhancer regulation. Nat Genet 49: 289-295, 2017.

29. Nagarajan S, Rao SV, Sutton J, Cheeseman D, Dunn S, Papachristou EK, Prada JG, Couturier DL, Kumar S, Kishore K, et al: ARID1A influences HDAC1/BRD4 activity, intrinsic proliferative capacity and breast cancer treatment response. Nat Genet 52: 187-197, 2020.

30. Helming KC, Wang X, Wilson BG, Vazquez F, Haswell JR, Manchester HE, Kim Y, Kryukov GV, Ghandi M, Aguirre AJ, et al: ARID1B is a specific vulnerability in ARID1A-mutant cancers. Nat. Med 20: 251-254, 2014.

31. Chang L, Azzolin L, Di Biagio D, Zanconato F, Battilana G, Lucon Xiccato R, Aragona M, Giulitti S, Panciera T, Gandin A, et al: The SWI/SNF complex is a mechanoregulated inhibitor of YAP and TAZ. Nature 563: 265-269, 2018.

32. Ruijtenberg S and van den Heuvel S: G1/S inhibitors and the SWI/SNF complex control cell-cycle exit during muscle differentiation. Cell 162: 300-313, 2015.

33. Wang Q, Zhou Y, Rychahou P, Harris JW, Zaytseva YY, Liu J, Wang C, Weiss HL, Liu C, Lee EY and Evers BM: Deptor is a novel target of Wnt $/ \beta$-Catenin/c-Myc and contributes to colorectal cancer cell growth. Cancer Res 78: 3163-3175, 2018.

34. Lissanu Deribe Y, Sun Y, Terranova C, Khan F, MartinezLedesma J, Gay J, Gao G, Mullinax RA, Khor T, Feng N, et al: Mutations in the SWI/SNF complex induce a targetable dependence on oxidative phosphorylation in lung cancer. Nat Med 24: 1047-1057, 2018.

35. Tsurusaki Y, Okamoto N, Ohashi H, Kosho T, Imai Y, Hibi-Ko Y, Kaname T, Naritomi K, Kawame H, Wakui K, et al: Mutations affecting components of the SWI/SNF complex cause Coffin-Siris syndrome. Nat Genet 44: 376-378, 2012. 
36. Kim KH, Kim W, Howard TP, Vazquez F, Tsherniak A Wu JN, Wang W, Haswell JR, Walensky LD, Hahn WC, et al: SWI/SNF-mutant cancers depend on catalytic and non-catalytic activity of EZH2. Nat Med 21: 1491-1496, 2015.

37. Rogers HA, Sousa S, Salto C, Arenas E, Coyle B and Grundy RG: WNT/ $\beta$-catenin pathway activation in Myc immortalised cerebellar progenitor cells inhibits neuronal differentiation and generates tumours resembling medulloblastoma. Br J Cancer 107: 1144-1452, 2012

38. Patel R, Brzezinska EA, Repiscak P, Ahmad I, Mui E, Gao M, Blomme A, Harle V, Tan EH, Malviya G, et al: Activation of $\beta$-catenin cooperates with loss of pten to drive ar-independent castration-resistant prostate cancer. Cancer Res 80: 576-590, 2020.

39. Yu J, Wu SW and Wu WP: A tumor-suppressive microRNA, miRNA-485-5p, inhibits glioma cell proliferation and invasion by down-regulating TPD52L2. Am J Transl Res 9: 3336-3344, 2017.
40. Chen Q, Wang P, Fu Y, Liu X, Xu W, Wei J, Gao W, Jiang K, Wu J and Miao Y: MicroRNA-217 inhibits cell proliferation, invasion and migration by targeting Tpd5212 in human pancreatic adenocarcinoma. Oncol Rep 38: 3567-3573, 2017.

41. Xu J, Wang W, Zhu Z, Wei Z, Yang D and Cai Q: Tumor protein D52-like 2 accelerates gastric cancer cell proliferation in vitro. Cancer Biother Radiopharm 30: 111-116, 2015.

42. Zhou J, Lin Y, Shi H, Huo K and Li Y: hABCF3, a TPD52L2 interacting partner, enhances the proliferation of human liver cancer cell lines in vitro. Mol Biol Rep 40: 5759-5767, 2013. International (CC BY-NC-ND 4.0) License. 